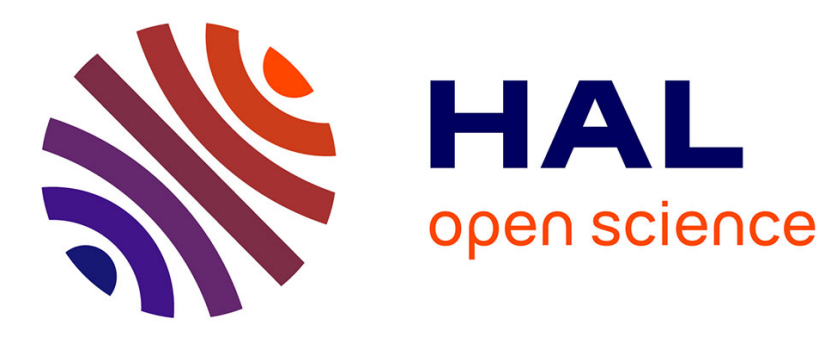

\title{
On the Informational Comparison of Qualitative Fuzzy Measures
}

\author{
Didier Dubois, Henri Prade, Agnès Rico
}

\section{To cite this version:}

Didier Dubois, Henri Prade, Agnès Rico. On the Informational Comparison of Qualitative Fuzzy Measures. 15th International Conference, Information Processing and Management of Uncertainty in Knowledge-based Systems (IPMU 2014), Jul 2014, Montpellier, France. pp.216-225, 10.1007/978-3319-08795-5_23. hal-01147279

\section{HAL Id: hal-01147279 https://hal.science/hal-01147279}

Submitted on 30 Apr 2015

HAL is a multi-disciplinary open access archive for the deposit and dissemination of scientific research documents, whether they are published or not. The documents may come from teaching and research institutions in France or abroad, or from public or private research centers.
L'archive ouverte pluridisciplinaire HAL, est destinée au dépôt et à la diffusion de documents scientifiques de niveau recherche, publiés ou non, émanant des établissements d'enseignement et de recherche français ou étrangers, des laboratoires publics ou privés. 


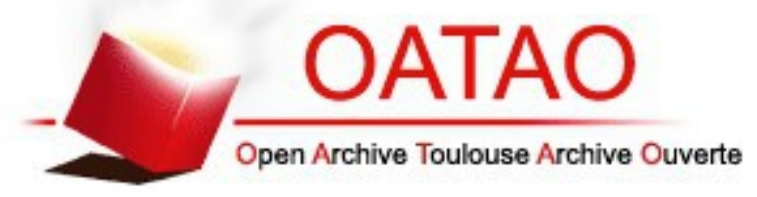

\section{Open Archive TOULOUSE Archive Ouverte (OATAO)}

OATAO is an open access repository that collects the work of Toulouse researchers and makes it freely available over the web where possible.

This is an author-deposited version published in : http://oatao.univ-toulouse.fr/ Eprints ID : 13203

To link to this article : DOI :10.1007/978-3-319-08795-5_23

URL : http://dx.doi.org/10.1007/978-3-319-08795-5_23

To cite this version : Dubois, Didier and Prade, Henri and Rico, Agnès On the Informational Comparison of Qualitative Fuzzy Measures. (2014) In: International Conference, Information Processing and Management of Uncertainty in Knowledge-based Systems - IPMU 2014, 15 July 2014 - 19 July 2014 (Montpellier, France).

Any correspondance concerning this service should be sent to the repository administrator: staff-oatao@listes-diff.inp-toulouse.fr 


\title{
On the informational comparison of qualitative fuzzy measures
}

\author{
Didier Dubois ${ }^{1}$ Henri Prade ${ }^{1}$ Agnès Rico $^{2}$ \\ 1 IRIT, CNRS and Université de Toulouse, France, \{dubois, prade\}@irit.fr \\ ${ }^{2}$ ERIC, Université de Lyon, France, agnes.rico@univ-lyon1.fr
}

\begin{abstract}
Fuzzy measures or capacities are the most general representation of uncertainty functions. However, this general class has been little explored from the point of view of its information content, when degrees of uncertainty are not supposed to be numerical, and belong to a finite qualitative scale, except in the case of possibility or necessity measures. The thrust of the paper is to define an ordering relation on the set of qualitative capacities expressing the idea that one is more informative than another, in agreement with the possibilistic notion of relative specificity. To this aim, we show that the class of qualitative capacities can be partitioned into equivalence classes of functions containing the same amount of information. They only differ by the underlying epistemic attitude such as pessimism or optimism. A meaningful information ordering between capacities can be defined on the basis of the most pessimistic (resp. optimistic) representatives of their equivalence classes. It is shown that, while qualitative capacities bear strong similarities to belief functions, such an analogy can be misleading when it comes to information content.
\end{abstract}

Keywords: Fuzzy measures, possibility theory, qualitative reasoning, information content

\section{Introduction}

Qualitative fuzzy measures (or q-capacities) are monotonic set-functions on a finite set with a range in a finite totally ordered set. They are instrumental in the representation of uncertainty of events, or yet, of the relative weights of groups of criteria in multicriteria evaluation, in the non-numerical environment, when likelihood or value scales are just totally or partially ordered sets, e.g. complete lattices [6]. An important issue to be clarified, if q-capacites are to be seriously considered as a tool for representing uncertainty, is the one of information content, and more generally the comparison of q-capacities in terms of their relative information content.

Important special cases of q-capacities are possibility and necessity measures [4]. For the latter set-functions, there is an abundant literature concerning information comparison, based on the notion of relative specificity $[15,4]$. Namely, a possibility (or a necessity) measure is entirely characterized by a possibility 
distribution over the elementary events, that defines a fuzzy set, and information comparison is carried out using fuzzy set inclusion, whereby a fuzzy set is more specific than another one, if and only if the former is a subset of the latter. However, there is almost no literature on the informational comparison of q-capacities in the general case. Some authors [11] have proposed notions of entropy for discrete capacities that evaluate the diversity of the coefficients appearing in the set-function. Our aim is rather to extend the specificity ordering to general q-capacities.

To do so, one may get inspiration from the theory of belief functions, where several informational orderings of various strength have been defined [14,3]. A belief function is a $\infty$-monotone set-function, that can be defined by means of a probability distribution over a power set (its Moebius transform), the degree of belief of an event summing the probability weights of all subevents that imply it. A belief function is then more informative than another if the former is eventwise greater than the latter. Another stronger definition, called specialisation, is based on the inclusion of focal sets. In the qualitative setting, it is also possible to compare q-capacities in an eventwise way. Besides, a qualitative counterpart of the Moebius transform can be defined, and a qualitative form of specialisation can be defined as well [13]. However, when indistinctly applied to q-capacites (like possibility and necessity measures), these definitions, even if formally welldefined, seem to baffle intuition.

In this paper, we try to provide a meaningful notion of information comparison between capacities, noticing that q-capacities differ not only by their information content, but also by the range of attitudes towards uncertainty they may encode. For instance, based on a given possibility distribution, the possibility measure is optimistic and the necessity measure is pessimistic [9], but one may argue they have the same information content.

\section{Framework and notations}

We consider a finite set (of states, criteria, etc.) $S=\left\{s_{1}, \cdots, s_{n}\right\}$ and a finite totally ordered scale $L$ with top 1 and bottom 0 . Let min denote the minimum, $\max$ the maximum. Moreover $L$ is equipped with an involutive negation $\nu$. A q-capacity is a mapping $\gamma: 2^{S} \rightarrow L$ such that $\gamma(\emptyset)=0, \gamma(S)=1$, and if $A \subseteq B$ then $\gamma(A) \leq \gamma(B)$. When $L=\{0,1\}, \gamma$ is called a Boolean capacity.

A special case of capacity is a possibility measure defined with a possibility distribution $\pi: S \rightarrow L$. The possibility measure is defined by $\Pi(A)=$ $\max _{s \in A} \pi(s)$. The value $\pi(s)$ is understood as the possibility that $s$ be the actual state of the world. Precise information corresponds to the situation where $\exists s^{*}, \pi\left(s^{*}\right)=1$ and $\forall s \neq s^{*}, \pi(s)=0$. Complete ignorance is represented by the vacuous possibility distribution $\pi^{\text {? }}$ such that $\forall s \in S, \pi^{?}(s)=1$. A possibility distribution $\pi$ is more specific than another possibility distribution $\rho$ if $\forall s \in S, \pi(s) \leq \rho(s)[15,4]$. This definition makes perfect sense since the set of possible values represented by $\pi$ is smaller, hence more precise, than the one represented by $\rho$. 
The q-capacity conjugate of $\gamma$, denoted by $\gamma^{c}$, is defined using the involutive negation $\nu$ by $\gamma^{c}(A)=\nu\left(\gamma\left(A^{c}\right)\right) \forall A \subseteq S$, where $A^{c}$ is the complement of the set $A$. In particular, the conjugate of a possibility measure is a necessity measure which is of the form $N(A)=\nu\left(\max _{s \notin A} \pi(s)\right)$.

The inner qualitative Moebius transform of $\gamma$ is a mapping $\gamma_{\#}: 2^{S} \rightarrow L$ defined by $\gamma_{\#}(E)=\gamma(E)$ if $\gamma(E)>\max _{B \subset E} \gamma(B)$ and 0 otherwise [10,12]. It contains the minimal information that is sufficient to reconstruct $\gamma$ as $\gamma(A)=$ $\max _{E \subseteq A} \gamma_{\#}(E)$. Let $\mathcal{F}^{\gamma}=\left\{E, \gamma_{\#}(E)>0\right\}$ denote the family of so-called focal sets associated to $\gamma$. Note that the inner qualitative Moebius transform of $\gamma$ is strictly monotonic with inclusion on $\mathcal{F}^{\gamma}$. The inner qualitative Moebius transforms of a possibility measure $\Pi$ coincides with its possibility distribution $\pi$ (focal sets are singletons) while the focal sets of a necessity measure $N$ are nested (the cuts of $\pi$ ).

\section{Can we transfer quantitative definitions of information comparison to the qualitative setting?}

When comparing capacities $\gamma$ and $\delta$, the inequality $\gamma \leq \delta$ is not always expressing something relevant about how informative $\gamma$ is with respect to $\delta$. Indeed, for instance if $\gamma$ is the vacuous possibility function $\Pi^{?}(A)=1$ if $A \neq \emptyset$ and $\delta$ is the vacuous necessity function $N^{?}(A)=0$ if $A \neq \Omega$, we have $\Pi^{?}>N^{?}$. However, they have exactly the same information content since based on the vacuous possibility distribution assigning 1 to all elements of $S$ and they are maximally uniformative among other possibility and necessity functions.

In the numerical setting, information comparison relations exist especially in the setting of belief functions. A belief function on $S$ is defined by means of a probability distribution $m$ over $2^{S} \backslash\{\emptyset\}$ as

$$
\operatorname{Bel}(A)=\sum_{E, E \subseteq A} m(E), \forall A \subseteq S .
$$

The conjugate functions are plausibility measures defined by $\operatorname{Pl}(A)=1-$ $\operatorname{Bel}\left(A^{c}\right) . \operatorname{Bel}(A)=1$ expresses full certainty of $A$, since then $\operatorname{Pl}\left(A^{c}\right)=0$ expresses that the complement $A^{c}$ is impossible.

There are several definitions of information comparison for belief functions. A belief function $B e l_{1}$ is said to be more informative (in the wide sense) than $\mathrm{Bel}_{2}$ if $\forall A \subseteq S, \operatorname{Bel}_{1}(A) \geq \operatorname{Bel}_{2}(A)$. This is due to the fact that $B e l_{1}$ assigns greater degrees of certainty to events (while $\operatorname{Bel}(A)=0$ expresses no information). In terms of imprecise probabilities, it is equivalent to have the inclusion $\{P$ : $\left.P \geq B e l_{1}\right\} \subseteq\left\{P: P \geq B \mathrm{Bel}_{2}\right\}$. And notice that for plausibility functions the inequality is reversed $\left(P l_{1}(A) \leq P l_{2}(A)\right)$.

Another information comparison method is based on specialisation, that relies on the mass assignments : $m_{1}$ is a specialization of $m_{2}$ (denoted by $m_{1} \sqsubseteq s m_{2}$ ) if and only if there exists a joint mass $x(A, B)$ with marginals $m_{1}$ and $m_{2}$, such that $x(A, B)=0$ whenever $A \nsubseteq B, A \in \mathcal{F}_{1}, B \in \mathcal{F}_{2}$. It expresses inclusion between 
focal sets of $B e l_{1}$ and $B e l_{2}$, that is sets $E$ with $m_{i}(E)>0$. It can be checked that $m_{1} \sqsubseteq_{s} m_{2}$ implies that $B e l_{1}$ is more informative (in the wide sense) than $\mathrm{Bel}_{2}$, but not conversely.

There is a formal analogy between q-capacities and belief functions, since $\gamma(A)=\max _{E, E \subseteq A} \gamma_{\#}(E)$, whereby $\gamma_{\#}$ plays the role of a mass assignment, and $\sum$ turns into max. This is why it is then tempting to consider $\gamma$ as more informative than $\delta$ whenever $\forall A \subseteq S, \gamma(A) \geq \delta(A)$. This is all the more natural as the following result, adapted from [13], holds:

Proposition 1. $\forall A \subseteq S, \gamma(A) \geq \delta(A)$ if and only if $\forall F \in \mathcal{F}^{\delta}, \exists E \in \mathcal{F}^{\gamma}$ s.t. $E \subseteq F, \gamma_{\#}(E) \geq \delta_{\#}(F)$.

Proof: $\gamma(A) \geq \delta(A)$ writes $\max _{E, E \subseteq A} \gamma_{\#}(E) \geq \max _{F, F \subseteq A} \delta_{\#}(F)$. Suppose $A=F$ is a focal set of $\delta$. Then the latter is equivalent to $\max _{E, E \subseteq F} \gamma_{\#}(E) \geq$ $\delta_{\#}(F)$, hence to $\forall F \in \mathcal{F}^{\delta}, \exists E \in \mathcal{F}^{\gamma}$ s.t. $E \subseteq F$ and $\gamma_{\#}(E) \geq \delta_{\#}(F)$.

Conversely, suppose the latter holds. If $\delta(\bar{A})=0$ then the result is obvious. If $\delta(A) \neq 0$ hence let us consider $F$ the focal element included in $A$ such that $\delta(A)=\delta_{\#}(F)$. There exists $E$ a focal element of $\gamma$ included in $F$ such that $\gamma_{\#}(E) \geq \delta(A)$. We have $E \subseteq F \subseteq A$ so $\gamma(A) \geq \gamma_{\#}(E) \geq \delta(A)$.

The condition $\forall F \in \mathcal{F}^{\delta}, \exists E \in \mathcal{F}^{\gamma}$ s.t. $E \subseteq F, \gamma_{\#}(E) \geq \delta_{\#}(F)$ is clearly a qualitative rendering of the specialisation relation. It formally means that for any focal set $F$ of $\delta$ there is a more important and more precise focal subset of $\gamma$, that explains the domination of $\gamma$ over $\delta$. Here, the two definitions of informational comparison are equivalent, which departs from the quantitative case.

However, this result is misleading. Neither the values of their lower Moebius transforms $\gamma_{\#}$ and $\delta_{\#}$, nor the size of focal sets $A, B$ with $\gamma_{\#}(A)>0$ and $\delta_{\#}(B)>0$ tell us much on their information content. For instance, focal sets of the vacuous possibility measure $\Pi^{\text {? }}$ are all singletons, and the unique focal set of $N^{\text {? }}$ is the whole set $S$. Viewing $\gamma$ as a counterpart to belief functions w.r.t. $\gamma_{\#}$ is thus not appropriate.

In such a context this article focuses on a basic question: When does a qcapacity represent the idea of certainty like belief functions, when does it represent plausibility (the conjugate of a belief functions)? In other words, when it is uncertainty-averse or pessimistic ? When it is uncertainty-prone or optimistic?

\section{Optimistic and pessimistic q-capacities}

Given a possibility distribution $\pi$ the corresponding $\Pi$ is optimistic in the sense that $\Pi(\{s\})=1$ as soon as $s$ is fully possible (among other states); and its corresponding conjugate $N=\Pi^{c}$ is pessimistic, in particular $N(\{s\})=0, \forall s \in S$ as soon as two distinct elements in $S$ are fully possible. More generally, $N(A) \leq$ $\Pi(A), \forall A \subseteq S$ and more specifically $N(A)>0$ implies $\Pi(A)=1$. Finally, $N(A)=1$ expresses full certainty while $\Pi(A)=1$ just expresses a lack of surprise for the occurrence of $A$. Likewise, belief functions are pessimistic while 
their conjugate plausibility functions, which rely on the same mass function, are optimistic.

The above considerations motivate the following definition.

Definition 1. A q-capacity $\gamma$ is said to be pessimistic (resp. optimistic) if $\gamma \leq$ $\gamma^{c}\left(\right.$ resp. if $\left.\gamma \geq \gamma^{c}\right)$.

This definition first appears in [6] where a pessimistic (resp. optimistic) capacity is called uncertainty-averse (resp: uncertainty-prone). It is easy to see that:

- a q-capacity may be neither pessimistic nor optimistic. There may exist $A, B$ such that $\gamma(A)<\gamma^{c}(A)$ (pessimistic for $A$ ), and $\gamma(B)>\gamma^{c}(B)$ (optimistic for $B)$.

- a q-capacity may be both : $\gamma=\gamma^{c}$ is possible. For instance with Boolean q-capacities $(L=\{0,1\})$ on a space with $2 n+1$ elements, $\gamma_{n}(A)=1$ if $|A|>n$ and 0 otherwise.

To check if a capacity is pessimistic, it is enough to check the property $\gamma(A) \leq$ $\gamma^{c}(A)$ for focal sets. For if $A$ is not focal, then there is a focal set $E$ contained in $A$ such that $\gamma(A)=\gamma(E)$, and it is clear that if $\gamma(E) \leq \gamma^{c}(E)$ then $\gamma(A) \leq$ $\gamma^{c}(E) \leq \gamma^{c}(A)$, since $A \subseteq E$. This remark helps checking the pessimism of $\gamma$.

Proposition 2. The following properties hold:

- If $\gamma$ is pessimistic then $\gamma(A)=1$ implies $\gamma\left(A^{c}\right)=0$. For Boolean capacities, $\gamma$ is pessimistic if and only if $\min \left(\gamma(A), \gamma\left(A^{c}\right)\right)=0$.

- If $\gamma$ is optimistic then $\gamma(A)=0$ implies $\gamma\left(A^{c}\right)=1$. For Boolean capacities, $\gamma$ is optimistic if and only if $\max \left(\gamma(A), \gamma\left(A^{c}\right)\right)=1$.

Proof: Let us suppose $\gamma$ pessimistic and $\gamma(A)=1$. Then $\gamma^{c}(A)=1$, hence $\gamma\left(A^{c}\right)=0$. Conversely in the binary case, either $\gamma(A)=0$ and then $\gamma(A) \leq$ $\gamma^{c}(A)$, or $\gamma\left(A^{c}\right)=0$ then $\gamma^{c}(A)=1$ and $\gamma(A) \leq \gamma^{c}(A)$.

The proof for the optimistic case is similar.

Note that we find a result proved in [2]. In that paper, a preference relation between acts represented by functions is called strongly pessimistic (resp. optimistic) if and only if it is represented by a Sugeno integral with respect to a necessity measure (resp. possibility measure). In that paper, contrary to ours, a preference relation is called pessimistic (resp. optimistic) the relation is represented with a Sugeno integral with respect to capacity $\gamma \operatorname{such}$ that $\min \left(\gamma(A), \gamma\left(A^{c}\right)\right)=0$ (resp. $\left.\max \left(\gamma(A), \gamma\left(A^{c}\right)\right)=1\right)^{3}$. Here we shall respectively call such capacities strictly pessimistic (resp. optimistic). Strictly pessimistic capacities are indeed special cases of pessimistic ones:

Proposition 3. If a capacity is such that for all subsets $A, \min \left(\gamma(A), \gamma\left(A^{c}\right)\right)=$ 0 then it is pessimistic.

Proof: Suppose $\gamma$ is not pessimistic. Then $\exists A, \gamma(A)>\nu\left(\gamma\left(A^{c}\right)\right)$. Hence $\gamma(A)>0$, but then by assumption, $\gamma\left(A^{c}\right)=0$, hence $\gamma(A)>1$, which is impossible.

\footnotetext{
${ }^{3}$ In the case of Boolean functions, Sugeno integral reduces to a capacity.
} 
Considering a capacity $\gamma$, for each $\alpha>0 \in L$ we can define a Boolean capacity $\gamma^{\alpha}$ called its $\alpha$-cut, as follows: for all $A \subseteq S, \gamma^{\alpha}(A)$ is equal to 1 if $\gamma(A) \geq \alpha$ and 0 otherwise. Then, $\gamma=\max _{\alpha \in L} \min \left(\alpha, \gamma^{\alpha}\right), \mathcal{F}^{\gamma}=\cup_{\alpha \in L} \mathcal{F}^{\left(\gamma^{\alpha}\right)}$, and we can show:

Proposition 4. A capacity is strictly pessimistic if and only if $\forall \alpha>0 \in L, \gamma^{\alpha}$ is pessimistic.

Proof: : If $\exists A, \alpha>0: \min \left(\gamma(A), \gamma\left(A^{c}\right)\right)=\alpha$, then $\gamma^{\alpha}(A)=\gamma^{\alpha}\left(A^{c}\right)=1$ hence by Proposition $2, \gamma^{\alpha}$ is not pessimistic. The converse is obvious.

We can moreover describe the topological features of families of focal sets of pessimistic q-capacities:

Proposition 5. $\gamma$ is a pessimistic q-capacity if and only if any two focal sets $E$ and $F$ that have empty intersection satisfy $\gamma_{\#}(E) \leq \nu\left(\gamma_{\#}(F)\right)$.

Proof: $\gamma \leq \gamma^{c}$ if and only if $\forall E \in \mathcal{F}^{\gamma}, \gamma_{\#}(E) \leq \nu\left(\gamma\left(E^{c}\right)\right)$. But $\gamma\left(E^{c}\right)=$ $\max _{F: F \cap E=\emptyset} \gamma_{\#}(F)$. So $\gamma_{\#}(E) \leq \min _{F: F \cap E=\emptyset} \nu\left(\gamma_{\#}(F)\right)$.

The condition $\gamma_{\#}(F)>\nu\left(\gamma_{\#}(E)\right)$ that prevents disjoint focal sets means that weights $\gamma_{\#}(F), \gamma_{\#}(E)$ are both high enough. In particular, the focal sets $E$ of a pessimistic $\gamma$ such that $\gamma_{\#}(E)>\nu\left(\gamma_{\#}(E)\right)$ (i.e., weights of focal sets in the upper part of the value scale) intersect pairwisely. All focal sets intersect the focal set with weight 1 . And if the focal sets of a q-capacity all pairwisely intersect, then the q-capacity is pessimistic. This property is characteristic for Boolean capacities: $\gamma$ is a pessimistic Boolean capacity if and only if the intersection of two focal sets is not empty.

In the non-Boolean case, one may have disjoint focal sets with small enough weights inside or overlapping focal sets with high weights.

Example 1. Consider a q-capacity with 4 focal sets, $E, F, G_{1}, G_{2}$ such that $F \subseteq$ $E, G_{i} \cap E \neq \emptyset$ for $i=1,2$, and $F, G_{1}, G_{2}$ are disjoint (Fig. 1). Assume $\gamma_{\#}(E)=$ $1, \gamma_{\#}(F)=\alpha>\nu(\alpha)$, and $\gamma_{\#}\left(G_{i}\right)=\beta_{i}<\nu(\alpha)$. Then it is easy to check that $\gamma$ is pessimistic, since $\gamma^{c}(E)=1, \gamma^{c}(F)=\nu\left(\max \left(\beta_{1}, \beta_{2}\right)\right)>\alpha, \gamma^{c}\left(G_{i}\right)=\nu(\alpha)>\beta_{i}$.

Fig. 1. Focal sets of a pessimistic q-capacity

For optimistic capacities one gets dual results:

Proposition 6. Let $\gamma$ be an optimistic q-capacity. If $A$ is a focal set such that $\gamma(A) \neq 1$ then $A^{c}$ contains a focal set of $\gamma$.

Proof: Let $A$ be a focal set of $\gamma \cdot \gamma(A)=\alpha$ entails $\gamma\left(A^{c}\right) \geq \nu(\alpha)>0$.

So for each $A$ such that $\gamma_{\#}(A) \neq 1$ we can find another focal set $B$ such that $A \cap B=\emptyset$. It means that the focal sets of optimistic capacities tend to be disjoint while those of pessimistic capacities tend to overlap. The precise description of focal sets of optimistic capacities can benefit from the knowledge of the focal sets of pessimistic ones, as explained in [8]. 


\section{Entailment between capacities}

Based on the above considerations, it makes sense to consider the following definition:

Definition 2. If $\gamma_{1}$ and $\gamma_{2}$ are two pessimistic capacities then $\gamma_{1}$ is more informative than $\gamma_{2}$ if and only if $\gamma_{1} \geq \gamma_{2}$ (eventwise).

Indeed, if a pessimistic q-capacity $\gamma_{1}$ assigns a high value to event $A$, then it will assign a very small value to its complement. In particular $\gamma_{1}(A)=1$ implies $\gamma_{1}\left(A^{c}\right)=0\left(\right.$ since $\left.\nu\left(\gamma_{1}\left(A^{c}\right)\right)=1\right)$, so that this kind of capacity expresses the idea of certainty, while its conjugate expresses the notion of plausibility [5] and satisfies the opposite statement. Hence if $\gamma_{1}$ systematically assigns a certainty value higher than or equal to the one assigned by $\gamma_{2}$ to events, the former provides more information than the latter.

Note that by construction, $\gamma_{1} \geq \gamma_{2}$ is equivalent to

$$
\left[\gamma_{1}(A), \gamma_{1}^{c}(A)\right] \subseteq\left[\gamma_{2}(A), \gamma_{2}^{c}(A)\right], \forall A \subseteq S
$$

In fact $\gamma_{1}$ and its conjugate contain the same amount of information but differ by the attitude toward uncertainty. As pointed out in [5], the width of the interval $\left[\gamma_{1}(A), \gamma_{1}^{c}(A)\right]$ reflects the quantity of ignorance regarding event $A$, namely, for any pessimistic capacity

- $\gamma_{1}(A)=1\left(=\gamma_{1}^{c}(A)\right)$ expresses the certainty of $A$.

$-\gamma_{1}^{c}(A)=0\left(=\gamma_{1}(A)\right)$ expresses the impossibility of $A$.

$-\gamma_{1}^{c}(A)=1$ and $\gamma_{1}(A)=0$ expresses total ignorance regarding $A$.

Note that if the optimistic capacities are two possibility measures, this notion of relative information content reduces to the specificity ordering: $\Pi_{1}$ is more informative than $\Pi_{2}$ if and only if $\Pi_{1} \leq \Pi_{2}$ if and only if $\pi_{1}$ is more specific than $\pi_{2}$, i.e. $\pi_{1} \leq \pi_{2}$.

Given a capacity $\gamma$ one can derive its pessimistic and optimistic versions (respectively called assurance and opportunity functions by Yager [16]):

Definition 3. The pessimistic version of a q-capacity $\gamma$ is $\gamma_{*}(A)=\min \left(\gamma(A), \gamma^{c}(A)\right)$ and its optimistic version is $\gamma^{*}(A)=\max \left(\gamma(A), \gamma^{c}(A)\right)$.

Note that $\gamma_{*}$ and $\gamma^{*}$ are both q-capacities (monotonic increasing with inclusion), and, by construction, $\gamma^{*}$ is the $\nu$-conjugate of $\gamma_{*}\left(\right.$ i.e. $\left.\gamma^{*}=\gamma_{*}^{c}\right)$ So each has the same information content as the other.

In such a context we can introduce a relation $\approx$ between q-capacities expressing the idea of containing the same amount of information :

Definition 4. $\gamma$ and $\delta$ contain the same amount of information, denoted by $\gamma \approx \delta$ if and only if $\gamma^{*}=\delta^{*}$ and $\gamma_{*}=\delta_{*}$.

This is an equivalence relation on the set of $L$-valued q-capacities on $S$. Note that we have $\gamma_{*}=\delta_{*}$ if and only if $\gamma^{*}=\delta^{*}$; so we just need to consider one 
equality. If $\gamma_{*}=\delta_{*}$ it means that for all subsets $A$ of $S$, the sets of values $\left\{\gamma(A), \gamma\left(A^{c}\right)\right\}$ and $\left\{\delta(A), \delta\left(A^{c}\right)\right\}$ are equal. So for each event $A$ we must decide the attitude in front of uncertainty : pessimistic if we assign the least value, optimistic if we assign the greatest one (while respecting monotonicity).

Proposition 7. The equivalence class $\mathcal{C}_{\approx}(\gamma)$ of $\gamma$ is upper bounded by $\gamma^{*}$ and lower-bounded by $\gamma_{*}$.

Proof: $\min \left(\gamma_{*}, \gamma_{*}^{c}\right)=\gamma_{*}$ so $\gamma_{*} \in \mathcal{C}_{\approx}(\gamma)$ and $\gamma_{*} \geq \min _{\delta \in \mathcal{C}_{\approx}(\gamma)} \delta$. Moreover for all $\delta \in \mathcal{C}_{\approx}(\gamma)$ we have $\delta \geq \min \left(\delta, \delta^{c}\right)=\delta_{*}=\gamma_{*}$ which entails $\min _{\delta \in \mathcal{C}_{\approx}(\gamma)} \delta \geq \gamma_{*}$. So $\min _{\delta \in \mathcal{C} \approx(\gamma)} \delta=\gamma_{*}$. Similarly, we can prove that $\max _{\delta \in \mathcal{C} \approx(\gamma)} \delta=\gamma^{*}$.

If $\delta \in \mathcal{C}_{\approx}(\gamma)$ is a pessimistic q-capacity, then $\min \left(\delta, \delta^{c}\right)=\delta=\gamma_{*}$ so $\gamma_{*}$ is the unique pessimistic q-capacity in $\mathcal{C}_{\approx}(\gamma)$. Similarly one obtains that $\gamma^{*}$ is the unique optimistic q-capacity in $\mathcal{C}_{\approx}(\gamma)$. As a consequence, we must compare q-capacities in terms of their information content via a comparison of their equivalence classes, which justifies the following definition :

Definition 5. A q-capacity $\gamma$ is said to be to be more informative than a $q$ capacity $\delta$ in the wide sense if and only if $\gamma_{*} \geq \delta_{*}$.

Likewise we can compare two q-capacities in terms of their relative pessimism in front of uncertainty by means of another relation:

Definition 6. A q-capacity $\gamma$ is said to be to be less pessimistic than a q-capacity $\delta$ in the wide sense if and only if $\left\{A: \gamma(A)=\gamma_{*}(A)\right\} \subseteq\left\{A: \delta(A)=\delta_{*}(A)\right\}$.

These definitions completely disentangle the two aspects carried by q-capacities: namely the attitude in front of uncertainty and the information content, in agreement with possibilistic specificity.

\section{Particular cases}

We consider in fact extreme cases of q-capacities.

Complete ignorance We consider the q-capacity defined by $\gamma(S)=1$ and $\gamma(A)=0$ otherwise. This is nothing but the vacuous necessity measure $N^{\text {? }}$ whose conjugate is $\Pi^{\text {? }}$. Clearly, $N^{\text {? }}$ is pessimistic and and it is less informative than any capacity, since $\left[N^{?}(A), \Pi^{?}(A)\right]=[0,1]$ for $A \neq S, \emptyset$.

Precise information For each element $s_{i}$ in $S$ we define the following qcapacity $\sigma_{i}(A)=\left\{\begin{array}{l}1 \text { if } s_{i} \in A \\ 0 \text { otherwise }\end{array}\right.$. It represents the precise information $x=s_{i}$. Note that $\sigma_{i}=\sigma_{i}^{c}$ is self-conjugate, so we have $\sigma_{i *}=\sigma_{i}^{*}=\sigma_{i}$. Some obvious remarks concerning this familly of q-capacities:

- If we consider two q-capacities $\sigma_{i}$ and $\sigma_{j}$ with $s_{i} \neq s_{j}$ then neither of the two is more informative than the other. 
- $\sigma_{i}$ and $\sigma_{j}$ contain the same information if and only if $s_{i}=s_{j}$.

$-\mathcal{C}_{\approx}\left(\sigma_{i}\right)=\left\{\sigma_{i}\right\}$, which is obvious since it is self-conjugate.

- There is no pessimistic q-capacity $\gamma \neq \sigma_{i}$ such that $\gamma \geq \sigma_{i}$. Indeed $\sigma_{i}$ is selfconjugate and $\left[\sigma_{i *}(A), \sigma_{i}^{*}(A)\right]$ reduces to 0 or 1, i.e., cannot contain the nontrivial intervals $\left[\gamma_{*}(A), \gamma^{*}(A)\right]$. For each capacity $\gamma$, either the information content of $\gamma$ and $\sigma_{i}$ are incomparable or $\gamma$ is less informative than $\sigma_{i}$.

Self-conjugate q-capacities In the numerical setting the most well-known self-conjugate capacities are probability measures that cannot be defined here, but for the precise q-capacities $\sigma_{i}$. Self-conjugate capacities $\sigma$ are such that $\forall A \subseteq S, \sigma(A)=\nu\left(\sigma\left(A^{c}\right)\right)=\sigma^{c}(A)$. They are at the same time pessimistic and optimistic. It is then obvious that they are maximally specific: there is no capacity that is more informative than any $\sigma$ since the intervals $\left[\sigma_{*}(A), \sigma^{*}(A)\right]$ are reduced to points, hence cannot contain $\left[\gamma_{*}(A), \gamma^{*}(A)\right]$ for any $\gamma \neq \sigma$. A remarkable subclass of self-conjugate capacities are symmetric ones, where $\gamma(A)=\alpha_{|A|}$ only depend on the cardinality of $A, \alpha_{|A|}=\nu\left(\alpha_{|S \backslash A|}\right) \geq \alpha_{|S \backslash A|}$ if $|A| \geq|S| / 2$. They are completely defined by a strictly increasing sequence of $n=|S|$ coefficients $\alpha_{i}$ such that $\alpha_{i}=\nu\left(\alpha_{n-i+1}\right)$. Of course, the precise q-capacities are a subclass of self-conjugate ones since if $\sigma_{\#}\left(\left\{s_{i}\right\}\right)=1$ then $\mathcal{F}^{\sigma}=\left\{\left\{s_{i}\right\}\right\}$, i.e. $\sigma=\sigma_{i}$.

\section{Conclusion}

This paper has tried to define the information content of a capacity when the value scale is purely ordinal. We have exploited the fact that a qualitative capacity accounts for both uncertainty and attitude in front of it, as pointed out in $[6,16]$. We propose a mathematical definition of information content comparison irrespective of the attitude toward uncertainty, and a comparison of capacities in terms of pessimism, irrespective of how much uncertainty they express.

Some issues remain open, and especially the structure of the set of focal sets of optimistic capacities. Informally, the focal sets of a pessimistic capacity tend to overlap each other, while the focal sets of an optimistic capacity tend to be disjoint. And the larger the focal sets, the less informative the pessimistic capacity. A strict form of pessimism is when all focal sets intersect pairwisely; maximal pessimism is obtained for nested focal sets (necessity measures). Alternatively, maximal pessimism is obtained for disjoint focal sets (possibility measures); then the smaller the focal sets the less informative the optimistic capacity. It would also be interesting to deepen the analogy and the differences between the qualitative and the quantitative settings for the modeling of uncertainty aversion. These results may be useful to clarify the potential use of qualitative capacities in various areas where aggregation functions are useful, especially in multicriteria analysis and information fusion. For instance, in a multiple source problem, q-capacities can be useful to merge possibility distributions [1]; focal sets of the q-capacity can then be understood as possibly antagonistic points of view on information. 


\section{References}

1. Z. Assaghir, A. Napoli et. al. : Numerical information fusion: lattice of answers with supporting arguments. Int. Conf. Tools for A.I., Boca Raton, 621-628, 2011.

2. A. Chateauneuf, M. Grabisch, A. Rico: Modeling attitudes toward uncertainty through the use of Sugeno integral. J. Math. Economics, 44:1084-1099, 2008.

3. D. Dubois, H. Prade: A set-theoretic view of belief functions - Logical operations and approximation by fuzzy sets. Int. J. of General Systems, 12(3): 193-226, 1986.

4. D. Dubois, H. Prade: Qualitative possibility functions and integrals. In: E.Pap, Ed., Handbook of measure theory, Elsevier, Amsterdam, Vol. 2, 1469-1521, 2002.

5. D. Dubois, H. Prade: Formal representations of uncertainty. In: D. Bouyssou, D. Dubois, M. Pirlot, H. Prade (Eds.), Decision-making Process- Concepts and Methods, ISTE London \& Wiley, Chap. 3, 85-156, 2009.

6. D. Dubois, H. Prade, M. Roubens, R. Sabbadin, J.-L.Marichal: The use of the discrete Sugeno integral in decision-making: a survey. Int. J. of Uncertainty, Fuzziness and Knowledge-Based Systems, 9(5): 539-561, 2001.

7. D. Dubois, H. Prade, A. Rico: Qualitative capacities as imprecise possibilities. In: Symbolic and Quantitative Approaches to Reasoning with Uncertainty, LNCS, vol. 7958, Springer, 169-180. 2013.

8. D. Dubois, H. Prade, A. Rico: Qualitative Capacities as Imprecise Possibilities.European Conference on Symbolic and Quantitative Approaches to Reasoning with Uncertainty (ECSQARU 2013), Utrecht,, Linda Van der Gaag (Eds.), Springer, LNCS 7958, 169-180, 2013.

9. D. Dubois, H. Prade. R. Sabbadin: Decision theoretic foundations of qualitative possibility theory. European Journal of Operational Research, 128: 459-478, 2001.

10. M. Grabisch: On the representation of $k$-decomposable measures. Proc. 7 th IFSA world Congress, Prague, vol. 1, 478-483, 1997.

11. Marichal, J.-L., Roubens M.: Entropy of discrete fuzzy measures. Int. J. Uncert. Fuzziness, and Knowledge-based Systems, 8: 635-640, 2000.

12. R. Mesiar, $k$-order pan-discrete fuzzy measures, Proc. 7th IFSA world Congress, Prague, vol. 1, 488-490, 1997.

13. H. Prade, A. Rico: Possibilistic Evidence. In: Weiru Liu (Eds.), European Conference on Symbolic and Quantitative Approaches to Reasoning with Uncertainty (ECSQARU 2011), LNAI 6717, Springer, 713-724, 2011.

14. R.R. Yager: Entropy and specificity in a mathematical theory of evidence. International Journal of General Systems 9: 249-260, 1983.

15. R.R. Yager: On the specificity of a possibility distribution. Fuzzy Sets and Systems, 50: 279-292, 1992.

16. R.R. Yager: Measures of assurance and opportunity in modeling uncertain information. Int. J. Intell. Syst. 27(8): 776-797, 2012. 